\title{
Generalized quantum Rabi model with both one- and two-photon terms: A concise analytical study
}

\author{
You-Fei Xie ${ }^{1}$, Liwei Duan ${ }^{1}$, and Qing-Hu Chen ${ }^{1,2, *}$ \\ 1 Department of Physics, Zhejiang University, Hangzhou 310027, China \\ 2 Collaborative Innovation Center of Advanced Microstructures, Nanjing University, Nanjing 210093, China
}

(Dated: January 4, 2019)

\begin{abstract}
A generalized quantum Rabi Hamiltonian with both one- and two-photon terms has emerged in the circuit quantum electrodynamics system for a decade. The usual parity symmetry is broken naturally in the simultaneous presence of both couplings, which complicates analytical treatments, even in the rotating wave approximations. In this paper, we propose an adiabatic approximation to this generic model by using Bogoliubov operators, and obtain a very concise analytical solution for both eigenvalues and eigenstates. Although the adiabatic approximation is only exact in the vanishing limit of the qubit frequency, the results for some physical observables nevertheless agree well with the numerical ones in a wide parameter regime. In the rotating-wave approximations, we also derive an analytical eigensolution. Two dominant Rabi frequencies are found in the Rabi oscillations of this generalized model. We also apply the present analytical theory to the vacuum Rabi splitting. It is found that some new phenomena emerge just because of the presence of the additional two-photon coupling term.
\end{abstract}

PACS numbers: 03.65.Yz, 03.65.Ud, 71.27.+a, 71.38.k

\section{INTRODUCTION}

The quantum Rabi model (QRM) describes the most simple and at the same time the most important coupling between a continuous degree of freedom (a mode of the light field) and a discrete one (a two-level system or qubit) which is linear in the quadrature operators 1]. In addition, the nonlinear coupling appears naturally as an effective model for a three-level system when the third (off-resonant) state can be eliminated. The twophoton model has been proposed to apply to certain Rydberg atoms in superconducting microwave cavities [2, 3]. Recently, a realistic implementation of the two-photon QRM using trapped ions has been proposed [4]. Usually, the two-photon term is the secondary effect, and thus limited to the weak-to-moderate coupling regime typical for experimental setups within cavity or circuit quantum electrodynamics (QED) systems .

Here we study a natural generalization of the QRM which exhibits both linear and non-linear couplings between both constituents, i.e. the QRM with both oneand two-photon terms, with Hamiltonian

$H=\frac{\Delta}{2} \sigma_{z}+\omega a^{\dagger} a+\sigma_{x}\left\{g_{1}\left(a^{\dagger}+a\right)+g_{2}\left[\left(a^{\dagger}\right)^{2}+a^{2}\right]\right\}$,

where $\Delta$ and $\omega$ are respectively frequencies of qubit and cavity, $\sigma_{x, z}$ are Pauli matrices describing the two-level system, $a\left(a^{\dagger}\right)$ are the annihilation (creation) bosonic operators of the cavity mode, and $g_{1}\left(g_{2}\right)$ is the one-photon (two-photon) qubit-cavity coupling constant.

The generalized QRM [5] described by Eq. (10) has actually been realized in a flux qubit coupled to the plasma mode of its superconducting quantum interference device (SQUID) detector to reduce the dephasing rate [6, 7] in 2005. Both coupling strengths $g_{1}$ and $g_{2}$ depend on the SQUID biased current. Recently, Felicetti et al. proposed a different circuit QED setup where a dc-SQUID is inductively coupled to a flux qubit and a current bias is added to the SQUID [8]. Expanding the qubit-SQUID interaction up to the second order can yield both linear and nonlinear interaction terms, although their original intention is to implement the twophoton QRM by setting zero dc current biases. Most recently, Pedernales et al. suggested that a background of a $(1+1)$-dimensional black hole requires a QRM with one- and two-photon terms that can be implemented in a trapped ion for the quantum simulation of Dirac particles in curved spacetime [9]. So the QRM with both oneand two-photon couplings not only is a generic model in the circuit QED, but also has wide applications in the area of cross-disciplinary research.

Both the one-photon QRM [1] and two-photon QRM 10] have been studied extensively for a few decades (for a review, please refer to Refs. [11 13] ). The analytical exact solutions based on the well-defined transcendental function have been only found recently for onephoton [14 and two-photon [15] QRM. These solutions have stimulated extensive research interests in the exact solutions to the QRM with both one-photon term [16 21] and two-photon term [22 25]. Many analytical approximate but still very accurate results have been also given 26 33]. In some model parameter limits, the dynamics and quantum criticality have been also studied exactly [34 36 .

In the QRM with both linear and nonlinear couplings, the parity symmetry is broken naturally, and the analytical solution becomes more difficult, in contrast to the unmixed models. To the best of our knowledge, no analytical solutions to these mixed model are available to date. In this paper, we propose concise analytical solutions to this generic model, which may be used as a solid basis for further advanced analytical studies. On 
the other hand, it could be easily applied to the circuit QED experiments where the expansion of qubit-oscillator interaction in the second-order is not negligible. As a preliminary application, we study effects of the nonlinear coupling on the vacuum Rabi splitting [37] by using these analytical solutions.

The paper is structured as follows: In Sec. II, by Bogoliubov operators, we propose a general scheme to solve the QRM with both one- and two-photon couplings. In Sec. III, the adiabatic approximations are made and concise analytical solutions are derived. The analytical energy level and qubit population dynamics are then presented and analyzed in Sec. IV. Section V is devoted to an analytic analysis in the rotating-wave approximations (RWAs). The applications of the analytical theories both in the adiabatic approximations and the RWA to the vacuum Rabi splitting are given in Sec. VI. The last section contains some concluding remarks.

\section{OPERATOR TRANSFORMATIONS AND NUMERICALLY EXACT SCHEME}

Associated with unmixed QRMs are the conserved parity $\Pi_{1 p}=-\sigma_{z} \exp \left(i \pi a^{+} a\right)$ for one-photon [1] and
$\Pi_{2 p}=-\sigma_{z} \exp \left(\frac{i \pi}{2} a^{+} a\right)$ for two-photon [23] qubit-cavity interaction, such that $[H, \Pi]=0$. $\Pi$ has two eigenvalues $( \pm 1)$ for the one-photon QRM, while it has four eigenvalues $( \pm 1, \pm i)$ for the two-photon QRM, because one can partition the Hilbert space in two subspaces, each with an $\mathrm{SU}(1,1)$ symmetry for the field depending on the photonic number. So in the two unmixed models, the parity symmetry acting in the bosonic Hilbert space greatly facilitates the study even in the analytical analysis. However, in the present generalized model with both one- and two-photon interactions with the qubit, no such conserved parity is available, so the analytical study is still challenging.

$$
H=\left(\begin{array}{cc}
a^{\dagger} a+g_{1}\left(a^{\dagger}+a\right)+g_{2}\left[\left(a^{\dagger}\right)^{2}+a^{2}\right] & -\frac{\Delta}{2} \\
-\frac{\Delta}{2} & a^{\dagger} a-g_{1}\left(a^{\dagger}+a\right)-g_{2}\left[\left(a^{\dagger}\right)^{2}+a^{2}\right]
\end{array}\right)
$$

To obtain a clean manifold, we perform Bogoliubov transformation to the bosonic degree of freedom so that the first diagonal element in Hamiltonian matrix (2) only consists of a free transformed bosonic number operator. The new bosonic operator is introduced as

$$
A=u a+v a^{\dagger}+w, A^{\dagger}=u a^{\dagger}+v a+w
$$

while the corresponding number state would be

$$
|n\rangle_{A}=S(r) D^{\dagger}(w)|n\rangle
$$

where $|n\rangle$ is the number state in original Fock space, $r=$ $\operatorname{arccosh} u, S(r)$ is the squeezing operator, and $D(w)$ is the displaced operator

$$
S(r)=e^{\frac{r}{2}\left(a^{2}-a^{\dagger 2}\right)}, D(w)=e^{w\left(a^{\dagger}-a\right)} .
$$

If set

$$
u=\sqrt{\frac{1+\beta}{2 \beta}}, v=\sqrt{\frac{1-\beta}{2 \beta}}, w=\frac{u^{2}+v^{2}}{u+v} g_{1},
$$

with $\beta=\sqrt{1-4 g_{2}^{2}}$, we have a simple quadratic form of the first diagonal element as

$$
H_{11}=\frac{A^{\dagger} A-v^{2}-w^{2}}{u^{2}+v^{2}},
$$

In this section, we introduce a scheme to pave the way to the solutions. For convenience, we write a transformed Hamiltonian with a rotation around the $y$ axis by an angle $\frac{\pi}{2}$ in the matrix form in units of $\omega=1$, which eigenstates are just $|n\rangle_{A}$.

Similarly, we can introduce another operator $B$,

$$
B=u a-v a^{\dagger}+w^{\prime}, B^{\dagger}=u a^{\dagger}-v a+w^{\prime},
$$

with the corresponding number state

$$
|n\rangle_{B}=S^{\dagger}(r) D^{\dagger}\left(w^{\prime}\right)|n\rangle .
$$

A simple quadratic form of the second diagonal element would be achieved if set $w^{\prime}=\frac{u^{2}+v^{2}}{v-u} g_{1}$ :

$$
H_{22}=\frac{B^{\dagger} B-v^{2}-w^{\prime 2}}{u^{2}+v^{2}} .
$$

In terms of the Bogoliubov operators $A$ and $B$, the Hamiltonian can be written as

$$
H=\left(\begin{array}{cc}
\beta\left(A^{\dagger} A-v^{2}-w^{2}\right) & -\frac{\Delta}{2} \\
-\frac{\Delta}{2} & \beta\left(B^{\dagger} B-v^{2}-w^{\prime 2}\right)
\end{array}\right) .
$$

Because now only the number operators $A^{+} A$ and $B^{+} B$ are present, in principle, the wavefunction can be expanded in terms of the number states of these new operators as

$$
|\rangle=\left(\begin{array}{c}
\sum_{n=0}^{n=0} c_{n}|n\rangle_{A} \\
\sum_{n=0}^{d_{n}}|n\rangle_{B}
\end{array}\right)
$$


Note that here the Hilbert space can be decomposed into different $n$ manifolds spanned by the spin and oscillator basis of $|\uparrow\rangle|n\rangle_{A}$ and $|\downarrow\rangle|n\rangle_{B}$, where $|\uparrow\rangle(|\downarrow\rangle)$ denotes the upper (lower) states of the qubit. Inserting Eq. (9) into the Schrödinger equation, we have

$$
\begin{aligned}
& \beta\left(m-v^{2}-w^{2}\right) c_{m}-\frac{\Delta}{2} \sum_{n=0}^{N_{t r}} D_{m n} d_{n}=E c_{m}, \\
& \beta\left(m-v^{2}-w^{2}\right) d_{m}-\frac{\Delta}{2} \sum_{n=0}^{N_{t r}} D_{n m} c_{n}=E d_{m},
\end{aligned}
$$

where $N_{t r}$ is the truncation number,

$$
D_{m n}=\sqrt{n ! m ! \beta}(u v \beta)^{(m+n) / 2} \exp \left(\frac{-2 g_{1}^{2}}{\beta^{3}}\right) \sum_{i=0}^{\min (m, n)} \frac{(-1)^{(m-i) / 2}(u v)^{-i}}{i !(m-i) !(n-i) !} H_{m-i}\left(\frac{g_{1}(u-v)}{\beta^{3 / 2} \sqrt{-u v}}\right) H_{n-i}\left(\frac{-g_{1}(u+v)}{\beta^{3 / 2} \sqrt{u v}}\right) .
$$

Here $H_{n}(x)$ stands for the Hermite polynomials.

In principle, based on Eqs. (10) and (11), we can obtain exactly the spectra of the generalized QRM to any desired accuracy by increasing the truncation number of the summation. For the numerical data presented below, we typically select $N_{t r}=60$, and converging results with relative errors less than $10^{-6}$ are convincingly arrived at.

\section{ADIABATIC APPROXIMATIONS}

In this section, we turn to an analytical scheme. In the framework of Eqs. (10) and (11), analytical approximations can be performed systematically. As a zero-order approximation, we only consider transition between states in the same manifold spanned by $|\uparrow\rangle|m\rangle_{A}$ and $|\downarrow\rangle|m\rangle_{B}$, then we have

$$
\begin{aligned}
& \beta\left(m-v^{2}-w^{2}\right) c_{m}-\frac{\Delta}{2} d_{m} D_{m m}=E c_{m}, \\
& \beta\left(m-v^{2}-w^{\prime 2}\right) d_{m}-\frac{\Delta}{2} c_{m} D_{m m}=E d_{m} .
\end{aligned}
$$

Nonzero coefficients give the following equation

$$
\begin{aligned}
& {\left[E-\beta\left(m-v^{2}-w^{2}\right)\right]\left[E-\beta\left(m-v^{2}-w^{2}\right)\right]} \\
& -\frac{\Delta^{2}}{4} D_{m m}^{2}=0 .
\end{aligned}
$$

The eigenvalues are then easily given by

$$
\begin{aligned}
E_{m}^{ \pm}= & \beta\left(m-v^{2}\right)-\frac{g_{1}^{2}}{\beta^{2}} \\
& \pm \frac{1}{2} \sqrt{\beta^{2}\left(w^{2}-w^{2}\right)^{2}+\Delta^{2} D_{m m}^{2}} .
\end{aligned}
$$

The corresponding eigenstate is

$$
|m\rangle_{ \pm} \propto\left(\begin{array}{c}
\frac{1}{2} \Delta D_{m m}|m\rangle_{A} \\
{\left[\beta\left(m-v^{2}-w^{2}\right)-E_{m}^{ \pm}\right]|m\rangle_{B}}
\end{array}\right) .
$$

As noted from Eqs. (10) and (11), it is just the qubit frequency $\Delta$ that correlates the different manifolds. The zero-order approximate results Eqs. (12) and (13) should be exact for the model if the qubit frequency vanishes, because the transitions among any manifolds disappear automatically. In literature, this approximation is also called the adiabatic approximation [38], which works best for small qubit frequency and strong coupling. For large qubit frequency, and weak coupling, the high order approximation should be performed. Actually, we can further consider the transitions between more neighboring manifolds, and more complicated analytical results would be derived. In the recent circuit QED, since the qubit frequency is usually smaller than the frequency of oscillator and the oscillator-qubit interaction has entered the ultrastrong 39 41], even deep-strong-coupling regime [42], the adiabatic approximation should work well. The further high-order corrections are not discussed here, and left for future study.

In the applications to the realistic circuit QED system, we also need to consider the full qubit Hamiltonian $H=-\left(\epsilon \sigma_{z}+\Delta \sigma_{x}\right) / 2$, where $\epsilon$ is the static bias. The eigenenergy can be easily derived as

$$
\begin{aligned}
E_{m}^{ \pm}= & \beta\left(m-v^{2}\right)-\frac{g_{1}^{2}}{\beta^{2}} \\
& \pm \frac{1}{2} \sqrt{\left(\beta w^{2}-\beta w^{\prime 2}+\epsilon\right)^{2}+\Delta^{2} D_{m m}^{2}} .
\end{aligned}
$$

\section{ENERGY LEVELS AND DYNAMICS}

With the recent progress in technology, the onephoton coupling term has reached the ultrastrongcoupling regime $\left(g_{1} / \omega \approx 0.12\right)$ experimentally with superconducting flux qubits inductively coupled to superconducting resonators [39 41]. More recently, it has ac- 

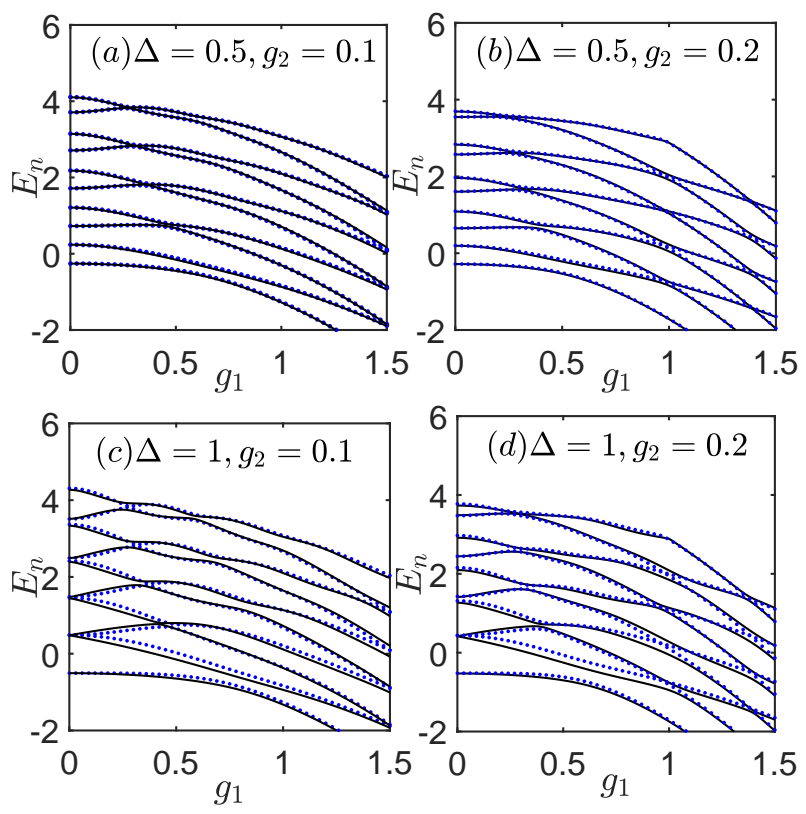

FIG. 1: (Color online) The energy levels (blue filled circles) as a function of $g_{1}$ for $\Delta=0.5, g_{2}=0.1$ and 0.2 (upper panel) and for $\Delta=1, g_{2}=0.1$ and 0.2 (lower panel). Numerical exact results are indicated by black solid lines.

cessed to the deep-strong-coupling regime $\left(g_{1} / \omega \approx 1.34\right)$ [42, 43]. The two-photon term emerges from the second process in cavity QED or the expansions of the qubitoscillator interaction up to the second order in the circuit QED, so the two-photon terms should not be strong generally. Physically, its dimensionless coupling strength $g_{2} / \omega$ should be less than the interaction-induced spectral collapse point 0.5 [4, 10, 23].

To show the validity of the adiabatic approximation to the recent experimentally accessible systems, we examine some physical observables such as the energy spectra and dynamics for the one-photon coupling strength $g_{1}$ ranging from weak to deep strong coupling, while the two-photon coupling strength $g_{2}$ is fixed to be a moderate value. The energy spectra can directly account for the experimental transmission spectrum, and dynamics can be measured experimentally.

The energy levels by Eq. (12) as a function of $g_{1}$ for $g_{2}=0.05$ and 0.1 , at the qubit frequency $\Delta=0.5$ and 1 are displayed in Fig. 1 1 . The numerically exact results by using Eqs. (10) and (11) are also collected. By the way, the exact results can be also obtained by the numerically diagonalization in original Fock space. For $\Delta=0.5$, the present results are in excellent agreement with the exact ones, while for $\Delta=1$ the adiabatic approximation still gives the good results. Actually, the present approach is basically a perturbation in the qubit frequency $\Delta$, so with increasing $\Delta$, the present results show a little bit deviation from the true spectra. Practically, in the recent experiments, $\Delta / \omega$ is usually not larger than 1 [39 42]. In

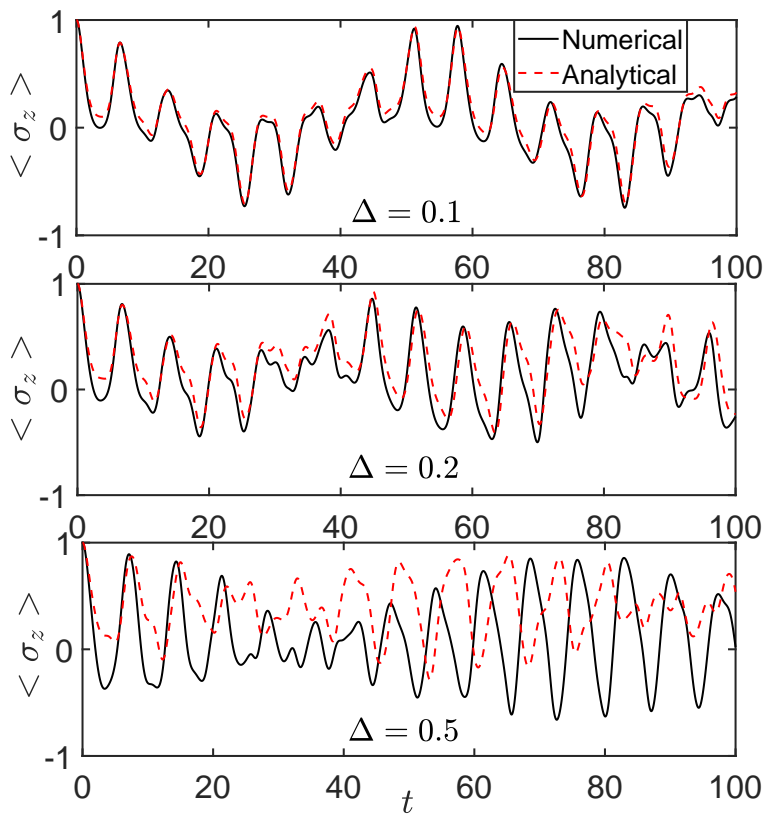

FIG. 2: (Color online) Population difference at $\Delta=0.1,0.2$ and 0.5 (from top to bottom) for $g_{1}=0.5, g_{2}=0.1$ initiated from photonic vacuum and upper level, which are denoted by red dashed lines. The numerical results are denoted by black solid lines.

the table I of Ref. [42], the value of $\Delta / \omega$ is even in the order of magnitude of 0.01 . In this sense, our results should be convincingly applicable to these circuit QED experiments if the two-photon coupling effect could be involved.

We then examine the dynamics of the population difference $\left\langle\sigma_{z}(t)\right\rangle$ for different qubit frequencies $\Delta=$ $0.1,0.2$, and 0.5 and the coupling strength $g_{1}=0.5, g_{2}=$ 0.1 in Fig. 2. For small value of $\Delta$, the adiabatic approximation can describe the dynamics almost exactly in the long time scale. For large $\Delta$, our analytical results can match the oscillation in phase for a long time. It only begins to get out of phase after many periods of oscillations. For all cases studied, our theory can basically give right description of the dynamics. Note that the parameters $\left(\Delta\right.$ and $\left.g_{1}\right)$ we used here are actually included but not limited to those in the recent experiments [39 42].

\section{ANALYTICAL ANALYSIS IN THE ROTATING-WAVE APPROXIMATIONS}

In the RWA, the one-photon QRM is reduced to Jaynes-Cummings model [44], which always reveals the general aspects of the full model at the weak coupling, such as Rabi oscillations, collapses and revivals of quantum state populations, entanglement dynamics, Schrödinger cat states.

Similar to the standard unmixed QRMs, the RWA can be made for the mixed model by neglecting the counter 
rotating terms, $g_{1}\left(a^{\dagger} \sigma_{+}+a \sigma_{-}\right), g_{2}\left[\left(a^{\dagger}\right)^{2} \sigma_{+}+a^{2} \sigma_{-}\right]$, which gives the Hamiltonian in the RWA as

$H=a^{\dagger} a+\frac{\Delta}{2} \sigma_{z}+g_{1}\left(a^{\dagger} \sigma_{-}+a \sigma_{+}\right)+g_{2}\left[\left(a^{\dagger}\right)^{2} \sigma_{-}+a^{2} \sigma_{+}\right]$.

It takes the following matrix form in the basis of $\sigma_{z}$

$$
H=\left(\begin{array}{cc}
a^{\dagger} a+\frac{\Delta}{2} & g_{1} a+g_{2} a^{2} \\
g_{1} a^{\dagger}+g_{2}\left(a^{\dagger}\right)^{2} & a^{\dagger} a-\frac{\Delta}{2}
\end{array}\right) .
$$

For one-photon case, the energy level for the QRM in the RWA reads [45]

$E_{n, 1 p}^{(k)}=n+\frac{1}{2}+(-1)^{k} \frac{1}{2} \sqrt{(\Delta-1)^{2}+4 g_{1}^{2}(n+1)}, k=1,2$.

with eigenfunctions consisting of $|n\rangle|\uparrow\rangle$ and $|n+1\rangle|\downarrow\rangle$. The RWA result for the eigenenergy for two-photon QRM [46] is given by

$E_{n, 2 p}^{(k)}=n+1+(-1)^{k} \frac{1}{2} \sqrt{(\Delta-2)^{2}+4 g_{2}^{2}(n+2)(n+1)}$,

with eigenfunctions consisting of $|n\rangle|\uparrow\rangle$ and $|n+2\rangle|\downarrow\rangle$.

For the case with both one- and two-photon couplings, we first propose the wavefunction as

$$
|n\rangle_{1}=\left(\begin{array}{c}
c_{n}|n\rangle \\
e_{n}|n+1\rangle+f_{n}|n+2\rangle
\end{array}\right), n=0,1,2, \ldots
$$

which includes the basic process of the two unmixed models. One should note that the Hamiltonian (15) cannot be decomposed cleanly into independent $n$ sub-space $R_{n}=\{|n\rangle|\uparrow\rangle,|n+1\rangle|\downarrow\rangle,|n+2\rangle|\downarrow\rangle\}$, because the interaction also couples the states in different subspaces, in sharp contrast to both unmixed models. So the true wavefunction should include the contribution from infinite bare states in all subspaces. This is to say, unlike the Jaynes-Cummings model, the mixed model in the RWA is not easy to solve. But it is still expected that the wavefunction (17) defined in the $n$ sub-space $R_{n}$ would be very accurate because dominant processes have been described.

By the Schrödinger equation, we can get an univariate cubic equation, which is given in detail in the appendix. Generally, there are three different real roots as listed in the end of the appendix. We observed that only the first root $\lambda_{1}$ from Eq. (A4) is closest to the numerical ones, which is denoted by $E_{n}^{(1)}$ for the generalized model. One can easily find that $E_{n}^{(1)}$ for $g_{2}=0\left(g_{1}=0\right)$ here is reduced to $E_{n, 1 p}^{(1)}\left(E_{n, 2 p}^{(1)}\right)$.

Both processes in the unmixed models can be also incorporated in another wavefunction

$$
|n\rangle_{2}=\left(\begin{array}{c}
f_{n}^{\prime}|n-1\rangle+c_{n}^{\prime}|n\rangle \\
e_{n}^{\prime}|n+1\rangle
\end{array}\right), n=0,1,2, \ldots
$$

Similarly, we can obtain another univariate cubic equation, which is also given in Appendix A. We find that only
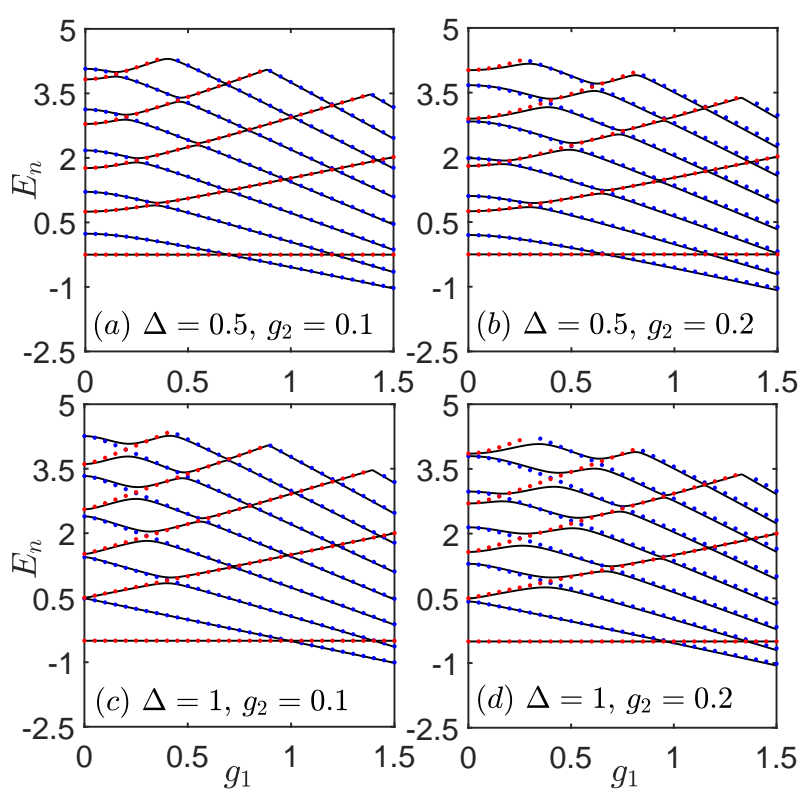

FIG. 3: (Color online) The energy levels as a function of $g_{1}$ for fixed $g_{2}$ for different $\Delta=0.5$ (upper panel) and 1 (lower panel). The numerical results are denoted by black solid lines. Blue and red dotted lines denote $E_{n}^{(1)}$ and $E_{n}^{(2)}$ respectively.

the third root $\lambda_{3}$ in Eq. (A6) is closest to the numerical ones, which is denoted by $E_{n}^{(2)}$ for the generalized model. Analogously, $E_{n}^{(2)}$ for $g_{2}=0\left(g_{1}=0\right)$ here is simplified to $E_{n, 1 p}^{(2)}\left(E_{n, 2 p}^{(2)}\right)$.

To show the validity of the approach with a few bare states, we compare the analytical results for the energy spectrum with the numerical ones. We also study the effect of moderate two-photon terms similar to the last section. The energy spectra as a function of $g_{1}$ with fixed $g_{2}=0.1,0.2$ for $\Delta=0.5$ and 1.0 are displayed in Fig. 3. It is interesting to note that the analytical results agree well with the numerical ones. Our result based on a few Fock states deviates from the exact ones slightly around the avoided crossings. In other words, around the avoided crossings, the contributions from the neighboring sub-spaces should be considered. It should be pointed out that the lowest red energy level in Fig. 3 can not be brought into the above general expression. Its energy is $-1 / 2$ with photonic vacuum states in atomic lower level.

Rabi oscillations.- With the eigensolutions obtained above, we can recheck many remarkable facets observed in the one-photon QRM in the RWA. Here we study the interplay effect of both couplings on the celebrated Rabi oscillation. Initiated from the number state in the upper level $|t=0\rangle=|n\rangle|\uparrow\rangle$, which can be expanded in terms of normalized eigenstates Eqs. (17) and (18)

$$
|n\rangle|\uparrow\rangle=d_{1}|n\rangle_{1}+d_{2}|n\rangle_{2}+d_{3}|n+1\rangle_{2},
$$


where

$$
d_{1}=c_{n}, d_{2}=c_{n}^{\prime}, d_{3}=f_{n+1}^{\prime},
$$

the time-dependent wavefunction is then determined as

$$
\begin{aligned}
& |\psi(t)\rangle=c_{n} e^{-i E_{n}^{(1)} t}|n\rangle_{1}+c_{n}^{\prime} e^{-i E_{n}^{(2)} t}|n\rangle_{2} \\
& +f_{n+1}^{\prime} e^{-i E_{n+1}^{(2)} t}|n+1\rangle_{2},
\end{aligned}
$$

we can get the photon states in the lower level

$$
\begin{aligned}
& |\psi(t), \downarrow\rangle=\left(c_{n} e^{-i E_{n}^{(1)} t} e_{n}+c_{n}^{\prime} e^{-i E_{n}^{(2)} t} e_{n}^{\prime}\right)|n+1\rangle \\
& +\left(c_{n} e^{-i E_{n}^{(1)} t} f_{n}+f_{n+1}^{\prime} e^{-i E_{n+1}^{(2)} t} e_{n+1}^{\prime}\right)|n+2\rangle .
\end{aligned}
$$

Then we have the population in the lower state

$$
\begin{aligned}
P_{\downarrow}= & c_{n}^{2}\left(1-c_{n}^{2}\right)+c_{n}^{\prime 2} e_{n}^{\prime 2}+f_{n+1}^{\prime 2} e_{n+1}^{\prime 2} \\
& +2 c_{n} e_{n} c_{n}^{\prime} e_{n}^{\prime} \cos \left[\left(E_{n}^{(1)}-E_{n}^{(2)}\right) t\right] \\
& +2 c_{n} f_{n} f_{n+1}^{\prime} e_{n+1}^{\prime} \cos \left[\left(E_{n}^{(1)}-E_{n+1}^{(2)}\right) t\right] .
\end{aligned}
$$

Finally we get qubit population difference $\left\langle\sigma_{z}\right\rangle=1-2 P_{\downarrow}$. Interestingly we obtain two Rabi frequencies: $\omega_{n}^{(1)}=$ $E_{n}^{(1)}-E_{n}^{(2)}, \omega_{n}^{(2)}=E_{n}^{(1)}-E_{n+1}^{(2)}$, unlike the unmixed model where only one Rabi frequency in the quantum Rabi oscillation is present. In the unmixed model with either one- or two-photon terms, $\omega_{n}^{(2)}$ disappears, so the evolution from a number state in the upper level oscillates sinusoidally.

We further examine the dynamics of the population difference $\left\langle\sigma_{z}(t)\right\rangle$ initiated from photonic vacuum states and qubit upper level at $\Delta=0.5$ for $g_{1}$ in the ultrastrongcoupling regime, and small values of $g_{2}$. For better understanding, we also analyze the Fourier transform of $\left\langle\sigma_{z}(t)\right\rangle$ in the long time window. The analytical results are shown in Fig. 4 with red lines. The numerical exact ones are also collected using black line for comparisons. The analytical results for $\left\langle\sigma_{z}(t)\right\rangle$ match quite well with the numerical ones. If $g_{1}$ and $g_{2}$ are comparable, two Rabi frequencies are clearly present with comparable peak height of the Fourier transform in the upper two panels for $g_{1}=0.1, g_{2}=0.05$ and 0.1. If $g_{1}$ and $g_{2}$ differ considerably, e.g. $g_{1}=0.5$, while $g_{2}=0.05$ and 0.1 , as demonstrated in the lower 2 panels, Fourier transform reveals a dominant oscillation with single frequency $\omega_{n}^{(1)}$ for $g_{1}>>g_{2}$.

Without the nonlinear coupling i.e. $g_{2}=0$, it is known that the famous Rabi oscillation with single frequency occurs. With the presence of the comparable nonlinear coupling, the two dominant oscillations are present. With the interplay of the one- and two-photon terms, population difference $\left\langle\sigma_{z}(t)\right\rangle$ becomes more complicated. If one kind of the coupling term is relatively weak, and therefore can be omitted, only one of the two quantum Rabi oscillations is visible.

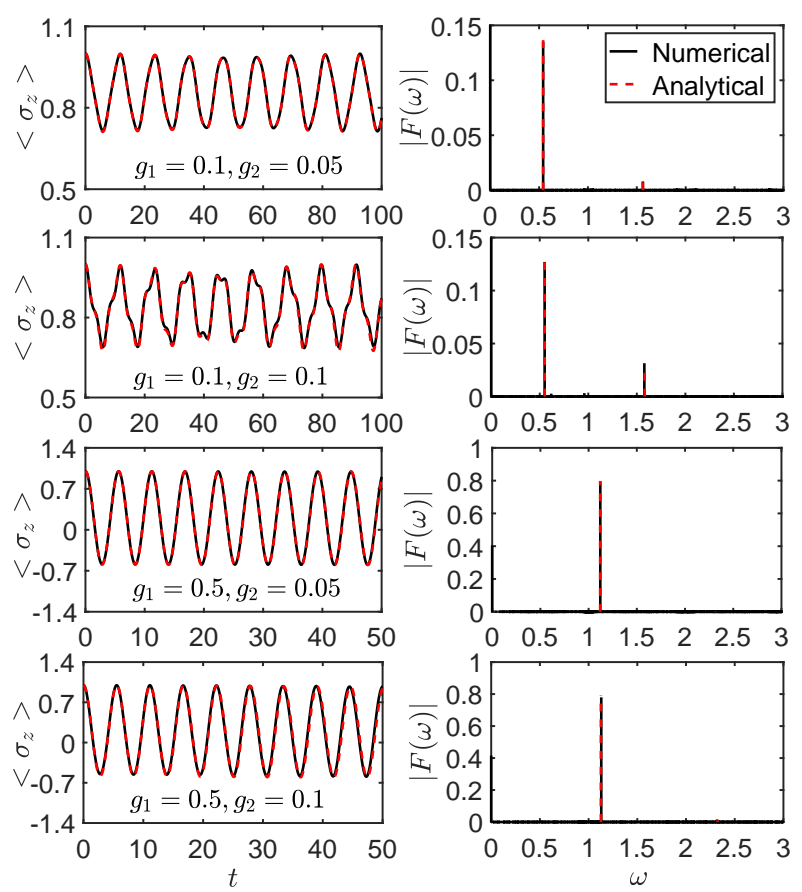

FIG. 4: (Color online) (left panel ) Population difference at $\Delta=0.5: g_{1}=0.1,0.5, g_{2}=0.05,0.1$ from top to bottom. (right panel ) The corresponding Fourier transform is calculated. The present analytical results and numerical ones are indicated by red and black lines.

Rabi oscillations are usually first measured in the newly-built superconducting qubit and a harmonic oscillator system [47] to demonstrate the strong coupling. The present emergent novel Rabi oscillations with two frequencies might be experimentally a signal of the two competitive couplings.

\section{VACUUM RABI SPLITTING}

In this section, we apply the analytical results for both the RWA and non-RWA cases derived above to the famous phenomenon of the vacuum Rabi splitting [37]. In the Jaynes-Cummings model, when an atom is pumped into an excited state with the vacuum photon state, $\left|\psi_{0}\right\rangle=|e\rangle|0\rangle$, it will decay to the ground state via spontaneous emissions. Note that the ground state of the atom-field system is just the direct product of the vacuum field and the ground-state atom. The two lowest excited eigenstates of the system are equivalently observed spectroscopically as a vacuum Rabi mode splitting resulting in the two-peak emission spectrum. The vacuum Rabi splitting was experimentally confirmed in many cavity [48] and circuit [49] QED systems.

In some proposed schemes to realize the qubit and oscillator coupling systems, the counter-rotating terms can be strongly suppressed [21, 50, 51]. In some devices, the anisotropy even appears quite naturally, because they are 


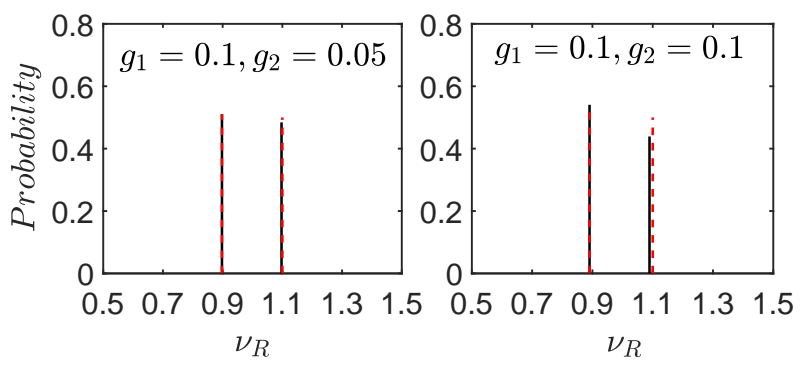

FIG. 5: (Color online) Emission spectrum $\nu_{R}$ in the RWA for initial state $|e\rangle|0\rangle$ at $g_{1}=0.1$ for $g_{2}=0.05$ (left) and $g_{2}=0.1$ (right) on resonance $(\Delta=1)$. The analytical results are indicated by red dashed lines and numerical exact results by black lines.

controlled by different input parameters [52]. Therefore it is technically feasible to realize the generalized QRM with and without the RWA.

In the present generalized QRM with the RWA, the initial states can be expanded in terms of states (17) and (18)

$$
\left|\psi_{0}\right\rangle=c_{0}|0\rangle_{1}+c_{0}^{\prime}|0\rangle_{2}+f_{1}^{\prime}|1\rangle_{2} .
$$

The probabilities to find the eigenstates $|0\rangle_{1},|0\rangle_{2},|1\rangle_{2}$ in the initial state are $c_{0}^{2}, c_{0}^{\prime 2}$, and $f_{1}^{\prime 2}$ respectively. The corresponding eigenvalues are $E_{0}^{(1)}, E_{0}^{(2)}$, and $E_{1}^{(2)}$. Then the atom can decay from these three states to the ground state and the emission spectrum obtained has in principle three peaks with height proportional to the corresponding probabilities and positions determined by the corresponding eigenenergies.

In Fig. 5, we plot the analytical emission spectrum at $g_{1}=0.1$ for $g_{2}=0.05$ and 0.1 on resonance $(\Delta=1)$ with red dashed lines. Both $g_{1}$ and $g_{2}$ are in the ultrasrongcoupling regime. The frequency is $\nu_{R}=E-E_{G S}$, where $E_{G S}=-\Delta / 2$ is the ground state energy. Practically, the spectrum has Lorentzian peaks due to the spontaneous emission to the ground state in the environment. Here the peaks are shown without width. The numerical results are also presented for comparison, and good agreement is demonstrated. In principle, three peaks should appear, but the height $\left(f_{1}^{\prime 2}\right)$ of the third peak is too small and omitted here. In the presence of the two-photon terms, the height of the second peak is lower than that of the first peak, and the peak difference becomes more pronounced with the two-photon coupling. Note that in the Jaynes-Cummings model, the emission spectrum has two peaks with equal probability on resonance. Without the two-photon coupling terms, the effects of counter-rotating terms on vacuum Rabi splitting have been studied with a coherent-state approach [53]. The spontaneous emission spectrum has multiple peaks, and the number of peaks increases with the coupling strength, in sharp contrast to vacuum Rabi splitting under the RWA.
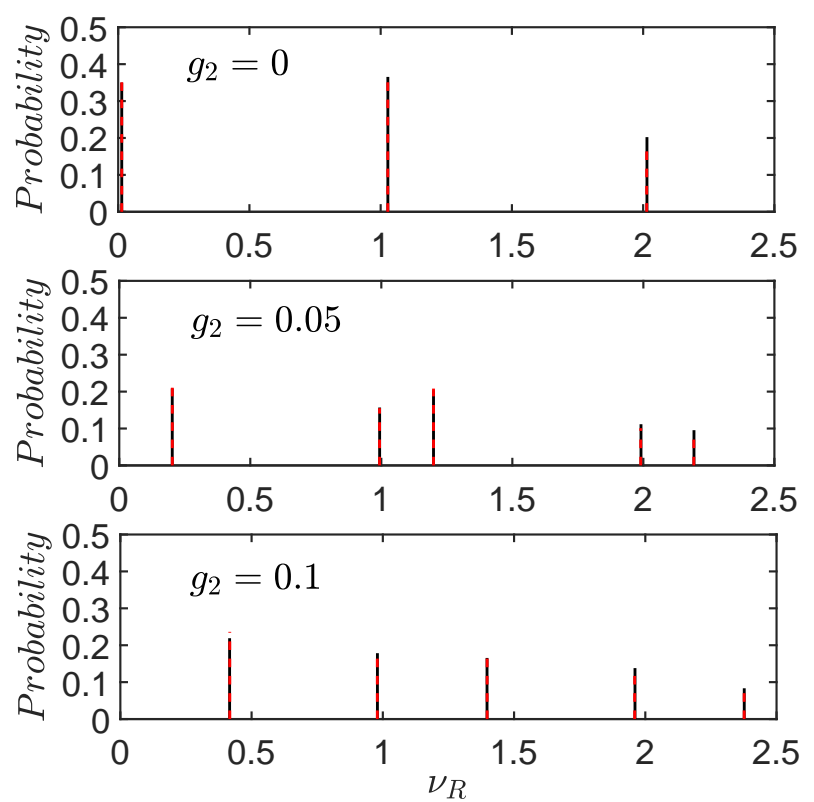

FIG. 6: (Color online) Emission spectrum $\nu_{R}$ without the RWA for initial state $|e\rangle|0\rangle$ at $\Delta=0.1, g_{1}=1$ for $g_{2}=0$ (top), 0.05 (middle), and $g_{2}=0.1$ (bottom). The analytical results are indicated by red dashed lines and numerical exact ones by black lines.

Now we study the effects of two-photon coupling on the vacuum Rabi splitting without the RWA. In this case, we will employ the analytical results (12) and (13) in the adiabatic approximation. In this case, we need to transform the wavefunction (13) to the original frame in the representation of $\sigma_{x}$. First, we write the normalized orthogonal wavefunction in the adiabatic approximation

$$
|m\rangle^{ \pm}=\left(\begin{array}{c}
c_{m}^{ \pm}|m\rangle_{A} \\
d_{m}^{ \pm}|m\rangle_{B}
\end{array}\right)
$$

transforming back to the original frame, we have

$$
\left|\varphi_{m}\right\rangle^{ \pm}=\frac{1}{\sqrt{2}}\left(\begin{array}{c}
c_{m}^{ \pm}|m\rangle_{A}-d_{m}^{ \pm}|m\rangle_{B} \\
c_{m}^{ \pm}|m\rangle_{A}+d_{m}^{ \pm}|m\rangle_{B}
\end{array}\right)
$$

According to these wavefunctions, the initial state can then be expanded as

$$
\begin{aligned}
\left|\psi_{0}\right\rangle= & \frac{1}{\sqrt{2}} \sum_{m=0}\left|\varphi_{m}\right\rangle^{-}\left(c_{m}^{-} D_{m}^{A}-d_{m}^{-} D_{m}^{B}\right) \\
& +\frac{1}{\sqrt{2}} \sum_{m=0}\left|\varphi_{m}\right\rangle^{+}\left(c_{m}^{+} D_{m}^{A}-d_{m}^{+} D_{m}^{B}\right),
\end{aligned}
$$


where

$$
\begin{aligned}
D_{m}^{A}= & \frac{1}{\sqrt{u m !}}\left(\frac{-v}{2 u}\right)^{m / 2} \exp \left(\frac{-w^{2}(u-v)}{2 u}\right) \\
& H_{m}\left(\frac{w(u-v)}{\sqrt{-2 u v})},\right. \\
D_{m}^{B}= & \frac{1}{\sqrt{u m !}}\left(\frac{v}{2 u}\right)^{m / 2} \exp \left(\frac{-w^{\prime 2}(u+v)}{2 u}\right) \\
& H_{m}\left(\frac{w^{\prime}(u+v)}{\sqrt{2 u v}}\right) .
\end{aligned}
$$

The probability to find the eigenstates $|m\rangle^{ \pm}$in the initial state is $P_{m}^{ \pm}=\frac{1}{2}\left(c_{m}^{ \pm} D_{m}^{A}-d_{m}^{ \pm} D_{m}^{B}\right)^{2}$.

We calculate the emission spectrum at $\Delta=0.1$, $g_{1}=1.0$ for three values of $g_{2}: 0,0.05$, and 0.1 in Fig. 6 both analytically and numerically. The frequency $\nu_{R}=E_{m}^{ \pm}-E_{0}^{-}$can be obtained with the use of Eq. (12). The analytical results are also in good agreements with the exact numerical results in this case. Compared to the unmixed one-photon QRM $\left(g_{2}=0\right)$, one can find that many more peaks in the spontaneous emission spectrum are induced when the two-photon coupling term sets in. We have confirmed this feature extensively with more model parameters.

Theoretically, it is found that some new phenomena emerge with the presence of the two-photon coupling terms, which may be the possible signal of the twophoton coupling besides the one-photon coupling in experiments.

\section{CONCLUSION}

In summary, the generalized QRM with both one- and two-photon terms is studied analytically. By using Bogoliubov operators, an effective scheme to its solution is proposed. The adiabatic approximation, where only the transition within the same manifold is considered, produces the analytical eigenvalues and eigenstates in a concise way. Many physical phenomena can then be easily analyzed. The obtained energy spectra, which can account for the experimental transmission spectrum, are in good agreement with the numerically exact ones in a wide range of coupling strength. The population dynamics obtained in the adiabatic approximation is also in quantitative agreement with the numerical ones.

In the RWA, the mathematical simplicity of the eigensolution in the unmixed Rabi models with either one- or two-photon term is lacking, because of the absence of the conserved excitation number. We propose an ansatz of the eigenfunctions including a few dominant Fock states. The corresponding analytical eigensolutions yield quite good energy levels compared with the numerical exact ones. With these eigensolutions, we could revisit many physical problems that have been studied in the unmixed QRM in the RWA. We study Rabi oscillations here. It is found that the population dynamics can also match the oscillations for a long time. Two dominant Rabi frequencies are derived analytically, and further confirmed in numerics.

The concise analytical solution in both full model and in the RWA can be easily applied in the circuit QED system with the one-photon coupling term ranging from weak, ultrastrong, to deep-strong-coupling regime for moderate two-photon coupling. Application of the analytical results to the vacuum Rabi splitting is performed in this paper as a example. In the RWA, the different heights of the first two peaks are observed. The second peak becomes lower with the increase of the twophoton coupling strength. Without the RWA, more peaks emerge with the advent of the two-photon coupling. These emergent new phenomena could be detected experimentally if both one- and two-photon interact with the oscillator simultaneously.

ACKNOWLEDGEMENTS This work is supported by the National Science Foundation of China (Nos. 11674285, 11834005), the National Key Research and Development Program of China (No. 2017YFA0303002).

* Corresponding author. Email:qhchen@zju.edu.cn

\section{Appendix A: Solutions in the rotating-wave} approximations by the univariate cubic equation

In terms of the wavefunction (17), the Schrödinger equation gives

$$
\begin{aligned}
& \left(n+\frac{\Delta}{2}-E\right) c_{n}|n\rangle+g_{1} e_{n} \sqrt{n+1}|n\rangle \\
& +f_{n} \sqrt{n+2}|n+1\rangle \\
& +g_{2}\left(e_{n} \sqrt{n(n+1)}|n-1\rangle+f_{n} \sqrt{(n+2)(n+1)}|n\rangle\right) \\
= & 0, \\
& g_{1} \sqrt{n+1}\left(c_{n}|n+1\rangle\right)+g_{2} \sqrt{(n+2)(n+1)} c_{n}|n+2\rangle \\
& +\left(n+1-\frac{\Delta}{2}-E\right) e_{n}|n+1\rangle \\
& +\left(n+2-\frac{\Delta}{2}-E\right) f_{n}|n+2\rangle \\
= & 0 .
\end{aligned}
$$

Note that the subspace that wavefunction spanned is not closed, unlike the unmixed model.

Projecting Eq. (A1) onto $|n\rangle$, Eq. (A2) onto $|n+1\rangle$ and $|n+2\rangle$, we have three set equations

$$
\begin{gathered}
\left(n+\frac{\Delta}{2}-E\right) c_{n}+g_{1} e_{n} \sqrt{n+1} \\
+g_{2} f_{n} \sqrt{(n+2)(n+1)}=0
\end{gathered}
$$




$$
\begin{array}{r}
g_{1} \sqrt{n+1} c_{n}+\left(n+1-\frac{\Delta}{2}-E\right) e_{n}=0, \\
g_{2} \sqrt{(n+2)(n+1)} c_{n}+\left(n+2-\frac{\Delta}{2}-E\right) f_{n}=0 .
\end{array}
$$

Set

$$
\begin{aligned}
& x=n+1-\frac{\Delta}{2}, \\
& y=g_{1} \sqrt{n+1}, \\
& z=g_{2} \sqrt{(n+2)(n+1)} .
\end{aligned}
$$

Nonzero coefficients yield a univariate cubic equation

$$
E^{3}+b E^{2}+c E+d=0
$$

where

$$
\begin{aligned}
& b=-3 x-\Delta, \\
& c=3 x^{2}+2 \Delta x-y^{2}-z^{2}+\Delta-1, \\
& d=x-x \Delta+x y^{2}+x z^{2}-x^{2} \Delta-x^{3}+y^{2} .
\end{aligned}
$$

Similarly by the other form of wavefunction Eq. (18), we can also obtain three set equations as follows

$$
\begin{gathered}
\left(n-1+\frac{\Delta}{2}-E\right) f_{n}^{\prime}+g_{2} \sqrt{(n+1) n} e_{n}^{\prime}=0, \\
\left(n+\frac{\Delta}{2}-E\right) c_{n}^{\prime}+g_{1} \sqrt{n+1} e_{n}^{\prime}=0, \\
g_{2} \sqrt{(n+1) n} f_{n}^{\prime}+g_{1} \sqrt{n+1} c_{n}^{\prime}+\left(n+1-\frac{\Delta}{2}-E\right) e_{n}^{\prime}=0 .
\end{gathered}
$$

set

$$
\begin{aligned}
x^{\prime} & =n+\frac{\Delta}{2}, \\
y^{\prime} & =g_{1} \sqrt{n+1}, \\
z^{\prime} & =g_{2} \sqrt{(n+1) n},
\end{aligned}
$$

we can obtain another cubic equation

$$
E^{3}+b^{\prime} E^{2}+c^{\prime} E+d^{\prime}=0
$$

where

$$
\begin{aligned}
& b^{\prime}=\Delta-3 x^{\prime} \\
& c^{\prime}=-y^{\prime 2}-z^{\prime 2}-1+3 x^{\prime 2}+\Delta-2 x^{\prime} \Delta \\
& d^{\prime}=-x^{\prime 3}-y^{\prime 2}+x^{\prime}\left(1+y^{\prime 2}+z^{\prime 2}-\Delta\right)+x^{\prime 2} \Delta,
\end{aligned}
$$

The solutions of the univariate cubic equation

$$
\lambda^{3}+b \lambda^{2}+c \lambda+d=0
$$

can be found in any Mathematics manual. If

with

$$
\Gamma=B^{2}-4 A C<0,
$$

$$
A=b^{2}-3 c, B=b c-9 d, C=c^{2}-3 b d,
$$

there are three different real roots

$$
\begin{aligned}
& \lambda_{1}=\frac{-b-2 \sqrt{A} \cos \theta}{3}, \\
& \lambda_{2}=\frac{-b+\sqrt{A}[\cos \theta-\sqrt{3} \sin \theta]}{3}, \\
& \lambda_{3}=\frac{-b+\sqrt{A}[\cos \theta+\sqrt{3} \sin \theta]}{3},
\end{aligned}
$$

where

$$
\theta=\frac{1}{3} \arccos \left(\frac{2 A b-3 B}{2 \sqrt{A^{3}}}\right)
$$

[1] D. Braak, Q.-H. Chen, M. Batchelor, and E. Solano, J. Phys. A: Math. Gen. 49, 300301 (2016).

[2] P. Bertet, S. Osnaghi, P. Milman, A. Auffeves, P. Maioli, M. Brune, J. M. Raimond, and S. Haroche, Phys. Rev. Lett. 88 143601(2002).

[3] M. Brune, J. M. Raimond, P. Goy, L. Davidovich, and S. Haroche, Phys. Rev. Lett. 59 1899(1987).

[4] S. Felicetti, J. S. Pedernales, I. L. Egusquiza, G. Romero, L. Lamata, D. Braak, and E. Solano, Phys. Rev. A 92 033817(2015).

[5] Z.-J. Ying, L. Cong, and X.-M. Sun, arXiv:1804.08128.

[6] P. Bertet, I. Chiorescu, C. J. P. M. Harmans, and J. E. Mooij, arXiv:cond-mat/0507290

[7] P. Bertet, I. Chiorescu, G. Burkard, K. Semba, C. J. P. M. Harmans, D. P. DiVincenzo, and J. E. Mooij, Phys. Rev. Lett. 95, 257002(2005).
[8] S. Felicetti, D. Z. Rossatto, E. Rico, E. Solano, and P. Forn-Díaz, Phys. Rev. A 97, 013851(2018).

[9] J. S. Pedernales, M. Beau, S. M. Pittman, I. L. Egusquiza, L. Lamata, E. Solano, A. del Campo, Phys. Rev. Lett. 120, 160403 (2018).

[10] K. M. Ng, C. F. Lo, and K. L. Liu, Eur. Phys. J. D 6 119 (1999)

[11] Q. -T Xie, H. -H Zhong, M. T. Batchelor, and C. -H. Lee, J. Phys. A 50, 113001(2016).

[12] X. Gu, A. F. Kockum, A. Miranowicz, Y.-x Liu, F. Nori, Physics Reports 718-719, 1-102 (2017).

[13] P. Forn-Díaz, L. Lamata, E. Rico, J. Kono, E. Solano, arXiv: $1804.09275 \mathrm{v} 1$.

[14] D. Braak, Phys. Rev. Lett. 107, 100401 (2011).

[15] Q. -H. Chen, C. Wang, S. He, T. Liu, and K. -L. Wang, Phys. Rev. A 86, 023822(2012). 
[16] H.-H. Zhong, Q.-T. Xie, M. T. Batchelor, and C.-H. Lee, J. Phys. A 46, 415302 (2013); ibid 47, 045301(2014).

[17] A. J. Maciejewski, M. Przybylska, T. Stachowiak, Phys. Lett. A 378, 3445(2014).

[18] S. A. Chilingaryan, B. M. Rodriguez-Lara, J. Phys. A 46, 335301(2013) .

[19] J. Peng, et al., J. Phys. A 47, 265303(2014) .

[20] H. Wang, S. He, L.W. Duan, Q. -H. Chen, EPL 106 , 54001 (2014) .

[21] Q. T. Xie, S. Cui, J. P. Cao, L. Amico, and H. Fan, Phys. Rev. X 4, 021046 (2014).

[22] I. Travěnec, Phys. Rev. A 85, 043805(2012), A. J. Maciejewski, M. Przybylska, and T. Stachowiak ibid. 91 037801(2015), I. Travěnec, ibid. 91, 037802(2015).

[23] L. W. Duan, Y.-F. Xie, D. Braak, Q.-H. Chen, J. Phys. A: Math. Theor. 49, 464002 (2016).

[24] Y. Z. Zhang, J. Math. Phys. 54, 102104 (2013); Annals of Physics 375, 460(2016).

[25] A.J. Maciejewski, T. Stachowiak, J. Phys. A: Math. Theor. 50, 244003 (2017).

[26] I. D. Feranchuk, L. I. Komarov, and A. P. Ulyanenkov, J. Phys. A 29, 4035 (1996).

[27] E. K. Irish, Phys. Rev. Lett. 99, 173601 (2007); erratum, Phys. Rev. Lett. 99, 259901 (2007).

[28] Q. -H. Chen, Y. Y. Zhang, T. Liu, and K. -L. Wang, Phys. Rev. A 78, 051801(R) (2008).

[29] C. J. Gan and H. Zheng, Eur. Phys. J. D 59, 473 (2010).

[30] S. He, C. Wang, Q.-H. Chen, X.-Z. Ren, T. Liu, and K.-L. Wang, Phys. Rev. A 86, 033837 (2012); S. He, Y. Zhao, and Q.-H. Chen, Phys. Rev. A 90, 053848 (2014).

[31] Z.-J. Ying, M. X. Liu, H.-G. Luo, H.-Q. Lin, J. Q. You, Phys. Rev. A 92, 053823 (2015)

[32] Y.-Y. Zhang, Phys. Rev. A 94, 063824 (2016).

[33] Z. G. Lv, C. J. Zhao, H. Zheng, J. Phys. A: Math. Theor. 50, 074002 (2017).

[34] J. Casanova, G. Romero, I. Lizuain, J. J. García-Ripoll and E. Solano, Phys. Rev. Lett. 105263603 (2010).

[35] M.-J. Hwang, R. Puebla, and M. B. Plenio, Phys. Rev. Lett.115, 180404 (2015).

[36] M. X. Liu, S. Chesi, Z.-J. Ying, X. S. Chen, H.-G. Luo, H.-Q. Lin, Phys. Rev. Lett. 119, 220601 (2017).
[37] J. J. Sanchez-Mondragon et al., Phys. Rev. Lett. 51, 550(1983); S. Agarwal, Phys. Rev. Lett. 53, 1732(1984).

[38] E. K. Irish, J. Gea-Banacloche, I. Martin, and K. C. Schwab, Phys. Rev. B 72, 195410 (2005).

[39] T. Niemczyk et al., Nature Physics 6, 772 (2010).

[40] P. Forn-Díaz et al., Phys. Rev. Lett. 105, 237001 (2010).

[41] Z. Chen, Y. M. Wang, T. F. Li, L. Tian, Y. Y. Qiu, K. Inomata, F. Yoshihara, S. Y. Han, F. Nori, J. S. Tsai, J. Q. You, Phys. Rev. A 96, 012325 (2017).

[42] F. Yoshihara, T. Fuse, S. Ashhab, K. Kakuyanagi, S. Saito, and K. Semba, Nat. Phys. 13, 44 (2016).

[43] P. Forn-Díaz, J. J. García-Ripoll, B. Peropadre, J.-L. Orgiazzi, M. A. Yurtalan, R. Belyansky, C. M. Wilson, and A. Lupascu, Nat. Phys. 13, 39 (2016).

[44] E. T. Jaynes and F. W. Cummings, Proc. IEEE 51, 89 (1963)

[45] M. O. Scully and M. S. Zubairy, Quantum Optics (Cambridge University Press, Cambridge, 1997); M. Orszag, Quantum Optics Including Noise Reduction, Trapped Ions, Quantum Trajectories, and Decoherence 2nd ed. (Springer, Berlin, 2007)..

[46] V. V. Albert, G. D. Scholes, and P. Brumer, Phys. Rev. A 84, 042110(2011).

[47] Chiorescu, I. et al. Nature 431, 159 (2004). Johansson, J. et al. Phys. Rev. Lett. 96, 127006 (2006).

[48] R. J.Thompson, G. Rempe, and H.J. Kimble, Phys. Rev. Lett. 68, 1132(1992).

[49] A. Wallraff et al., Nature 431, 162(2004); A. Boca et al., Phys. Rev. Lett. 93, 233603(2004); A. Abdumalikov et al., Phys. Rev. B 78, 180502 (2008); A. Fedorov, et al., Phys. Rev. Lett. 105, 060503(2010).

[50] J. Keeling, M. J. Bhaseen, and B. D. Simons, Phys. Rev. Lett. 105, 043001 (2010).

[51] A. Baksic and C. Ciuti, Phys. Rev. Lett. 112, 173601 (2014).

[52] A. L. Grimsmo and S. Parkins, Phys. Rev. A 87033814 (2013).

[53] Y.-Y. Zhang, Q.-H. Chen, and S.-Y. Zhu, Chin. Phys. Lett. 30, 114203 (2013). 